\title{
Genetic studies of quantitative variation in a component of human saliva
}

\author{
By J. D. NISWANDER, ${ }^{*} †$ D. C. SHREFFLER* AND J. V. NEEL*
}

For many years individual genetically controlled variation in the content of ABH and Lewis substance in human saliva has been recognized (Schiff \& Sasaki, 1932; Grubb, 1948). A number of recent studies have suggested considerable variability in other constituents of human saliva. Thus, Kostlin \& Rauch (1957), from the results of paper electrophoresis on human parotid and submandibular secretions, concluded that 'the protein fractions of similar glands differ from one subject to the next'. Immuno-electrophoresis of saliva has been carried out by Gabl \& Pastner (1960), who found a component with mobility similar to serum albumin whose concentration varied in the ten individuals studied. Patton \& Pigman (1959) found, by Tiselius electrophoresis and ultracentrifugation, that the number of components obtained from parotid and submaxillary saliva showed more constancy in successive samples from the same individual than for samples from different individuals. However, with the exception of the $\mathrm{ABH}$ and Lewis secretion systems, little is known concerning the role of genetic factors in this variability. Furthermore, the only work on experimental animals appears to be that of Schneyer (1958), who has reported data suggesting genetic control of salivary amylase levels in mice.

The present studies were undertaken on the premise that suitable techniques would demonstrate further variability in human saliva, and that genetic factors might be of importance in this variability. In this paper we will describe a specific component of saliva, detected by serological methods, which shows wide variation between individuals in its concentration. It will be referred to as $\mathrm{SC}_{1}$, indicating the salivary component migrating most anodally on electrophoresis. A quantitative immunodiffusion technique, a modification of methods described by Crowle (1961) and Hayward \& Augustine (1957), has been applied to a series of salivas from twins and from family members in an attempt to determine whether the level of this factor is under genetic control.

\section{MATERIALS AND METHODS}

This investigation was carried out in two phases-descriptive and exploratory studies, and genetic studies. In the first stage, attempts were made to identify and characterize those components exhibiting the greatest variation and to study possible non-genetic sources of this variation. Most of this work was conducted using samples collected 'at random' from clerical and laboratory personnel and their families. Approximately seventy-five individuals contributed both whole and parotid saliva samples. The latter were collected with the aid of a plastic cup placed over the orifice of Stenson's duct (Curby, 1953). All parotid saliva was obtained with stimulation by lemon juice. Parotid, rather than whole saliva, was used in much of this work to avoid complications due to known differences in the secretions of the major salivary glands, or to contamination of whole saliva with bacteria, food particles, cellular debris, etc. Subsequent work

* Department of Human Genetics, University of Michigan Medical School, Ann Arbor, Michigan.

$\uparrow$ Present address: Department of Health, Education, and Welfare, U.S. Public Health Service, National Institutes of Health, National Institute of Dental Research, Bethesda, Maryland. 
has shown that while definitive differences exist between whole saliva and parotid secretions, contamination by extraneous substances does not appear to be a major factor in these differences. A few parotid samples were obtained by cannulation to check for possible contamination by whole saliva of specimens collected with the Curby cup. No evidence of contamination was found. Another source of material for this phase of the study was a series of three whole saliva samples taken from each of forty-four adult females being studied by Dr George Solish with respect to the occurrence of isoagglutinins in the secretions of the uterine cervix.

For the genetic aspects of this investigation, a number of saliva specimens selected from material stored in the Department of Human Genetics at the University of Michigan Medical School were utilized as follows:

(1) Saliva samples from a group of 208 adult male twin pairs residing in the Detroit-Chicago area. The diagnosis of zygotic type was based on an examination of the ABO, MN, Rh, Kell, Duffy, Secretor, P, Haptoglobin, and Gm types, as well as a number of morphological characteristics (height, weight, eye and hair colour, hair form, and appearance in standardized photographs). Ninety-six of the pairs were monozygotic and 112 dizygotic.

(2) A group of whole saliva samples collected in the course of a study of possible associations between blood groups and certain pediatric diseases. From this material forty-four healthy, male, non-twin sib pairs were randomly selected.

(3) A group of 150 families selected on the basis of availability of samples from parents and two or more children. This group is part of a continuing health survey involving an entire midwestern town, undertaken by the Department of Epidemiology of the School of Public Health, for whose generous co-operation we are most grateful.

All whole saliva specimens were collected without stimulation, boiled for $15 \mathrm{~min}$, and centrifuged for $5 \mathrm{~min}$. Saliva specimens were concentrated, when necessary, by ultrafiltration. Total protein concentration was measured in selected samples by the biuret technique of Weichselbaum (1946). The samples were stored at $-20^{\circ} \mathrm{C}$.

The medium for immunodiffusion tests was 1.5\% Difco Bacto-Agar, $0.72 \% \mathrm{NaCl}, 0.6 \%$ sodium citrate, $0.01 \%$ merthiolate, $0.1 \%$ sodium azide, and $0.01 \%$ trypan blue, at $\mathrm{pH} 6.7$. The medium and method were essentially as described by Ridgway, Klontz \& Matsumoto (1962). Immuno-electrophoresis was carried out at 5-6 V./cm. in Ionagar (Consolidated Laboratories, Chicago), using a barbital-lactate buffer, as described by Hirschfeld (1959).

\section{Antisera}

The following antisera were prepared against individual or pooled human saliva:

No. 86-rabbit anti-whole saliva (D.S.).

No. 100-rabbit anti-whole pooled saliva.

No. 2-rabbit anti-parotid saliva (M.H.).

No. 4 rabbit anti-parotid saliva (D.S.).

No. 9-rabbit anti-parotid $\mathrm{SC}_{1}$ fraction (J.N.).

The same immunization schedule was followed for all rabbits.

(1) $0.75 \mathrm{ml}$. saliva plus $0.75 \mathrm{ml}$. Freund's complete adjuvant, intraperitoneally.

(2) Same, repeated 2 weeks later.

(3) $0.75 \mathrm{ml}$. saliva without adjuvant intraperitoneally, 2 weeks after injection 2. 


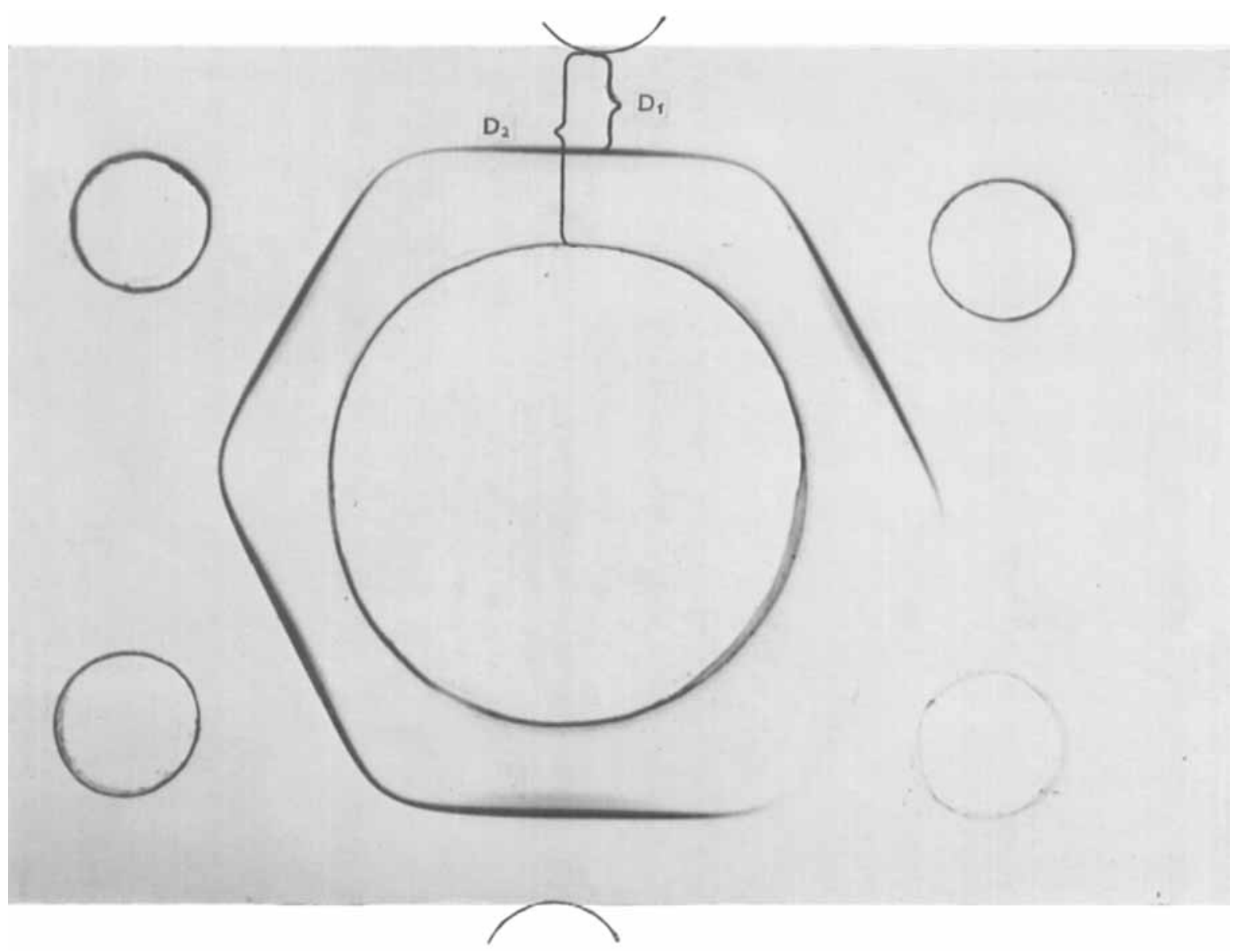

Immunodiffusion pattern illustrating measurements used for obtaining relative precipitin band position $\left(R={ }_{\mathrm{D}_{2}}^{\mathrm{D}_{2}}\right)$. Outer wells contain whole boiled saliva and inner wells contain anti-parotid saliva serum no. 4. 


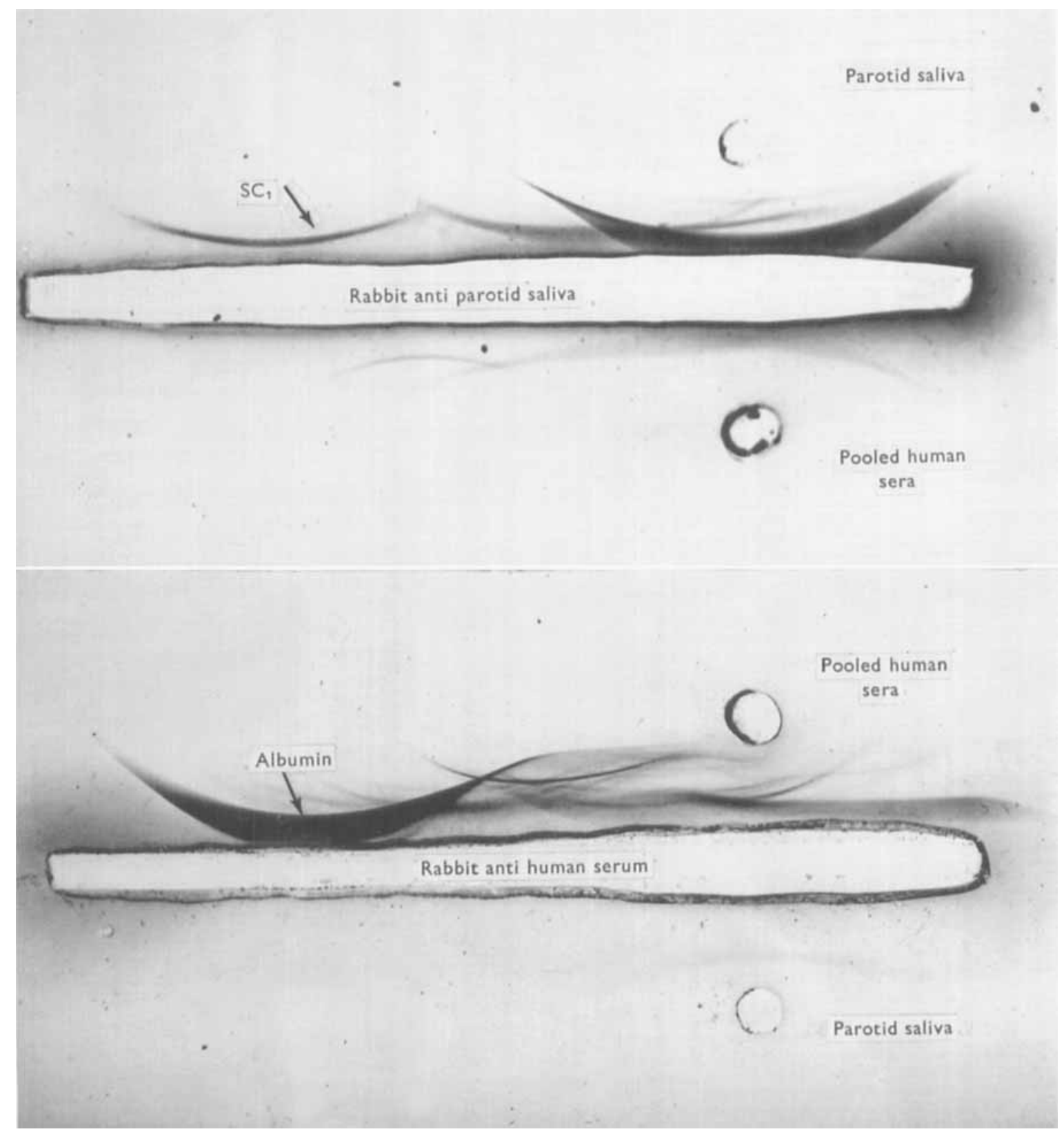

Immuno-electrophoretic patterns with antigen and antisera as noted. $\mathrm{SC}_{1}$ migrates toward the anode. Electrophoresis was at 5-6 V./cm. for $2 \mathrm{hr}$. Albumin and $\mathrm{SC}_{1}$ labelled for comparison of mobility. 
(4) $0.5 \mathrm{ml}$. saliva without adjuvant intravenously on day following injection 3 .

(5) Two bleedings of $40 \mathrm{ml}$. each on successive days, 1 week later.

(6) 3-5 were repeated one or more times, each time after a 6-week recovery period.

\section{Quantitative immunodiffusion method}

A measured quantity of agar, $11.5 \mathrm{ml}$, was poured on a $3 \frac{1}{4} \times 4$ in. glass plate to form a uniform layer, approximately $1.5 \mathrm{~mm}$. thick. Hexagonal patterns were cut in the gel using a standard agar gel cutter. Twelve $\lambda$ of saliva was placed in each of the outer wells and $115 \lambda$ of antiserum in the centre well. The plates were then placed in moist, sealed containers and incubated at $37^{\circ} \mathrm{C}$. for 18-20 hr. At constant volumes of antigen solutions, with incubation for a fixed time interval, the position of the precipitin band is determined by the relative concentration of antigen, i.e. the less concentrated the antigen, the closer to the antigen well the precipitate forms. The band position was read using a low power binocular microscope with a micrometer ocular. Distance was determined along a line between the centres of the wells and was measured from the edge of the antigen well to the outer edge of the precipitin band. The distance between antigen and antibody wells varied slightly, within patterns, and from plate to plate, probably due to distortion of the agar from cutting the pattern. It was therefore necessary to use the ratio of the distance between the antigen well and the precipitin band to the total distance between wells as the observed variable, rather than the direct reading of the band position (cf. Pl. 1).

Members of a twin or sib pair were always placed in adjacent wells of the same pattern to reduce errors due to variation in the amount of antiserum placed in the centre well or to slight differences in incubation time. However, twin pairs were tested without reference to zygotic type.

\section{RESULTS}

Immuno-electrophoretic patterns obtained from parotid saliva concentrated approximately ten volumes and from pooled human serum are shown in Pl. 2. The component to be considered here is shown by the arrow and is designated $\mathrm{SC}_{1}$. It will be noted that $\mathrm{SC}_{1}$ has a mobility similar to serum albumin. It has been determined, by inhibition and absorption experiments as well as by isolating $\mathrm{SC}_{1}$ and testing it against a specific anti-albumin, that this component is not serum albumin. Antiserum no. 4, which reacts strongly with $\mathrm{SC}_{1}$, gives no reaction against isolated serum albumin, although the same albumin preparation can be shown to react with antisera known to contain antibodies against albumin. Furthermore, inhibition of the rabbit antisaliva serum with various levels of pooled human serum has no effect on reactivity against $\mathrm{SC}_{1}$, indicating the absence of this component in serum. Using other antisera, e.g. no. 2, albumin could be detected in saliva. Albumin concentration appears to vary considerably from person to person, particularly in parotid saliva, but no systematic study of albumin variation has been undertaken.

A limited attempt was made to characterize the material. Boiling the saliva for $15 \mathrm{~min}$. did not diminish $\mathrm{SC}_{1}$ activity, although all but one other minor component were removed. Treatment with alcohol, acetone, or $15 \%$ TCA did not affect $\mathrm{SC}_{1}$. However, when boiled saliva was treated with $90 \%$ phenol and ethanol, a method used by Kabat (1956) for precipitation of mucopolysaccharide blood group substances, a heavy precipitate formed. The supernatant proved to have little or no $\mathrm{SC}_{1}$ activity, whereas the precipitate, which could be redissolved in alcohol or saline, showed marked $\mathrm{SC}_{1}$ activity. The $\mathrm{SC}_{1}$ band on the immuno-electrophoretic patterns did 
not react with stains for glycoproteins (Grabar, 1959), lipids (Grabar, 1959), or esterases (Uriel, 1961), or with Alcian Blue 8GX, an acid mucopolysaccharide stain (Heremans \& Vaerman, 1958). $\mathrm{SC}_{1}$ was separated by electrophoresis on agar and eluted from the gel. The eluate was spotted on filter paper along with control eluates from other areas of the electrophoretic pattern, and the paper was stained with Alcian Blue $8 \mathrm{GX}$. The $\mathrm{SC}_{1}$ spot showed significantly greater staining than the control spots. Immunodiffusion tests of the eluates revealed the presence of $\mathrm{SC}_{1}$ only in the most deeply staining fraction. The failure of the $\mathrm{SC}_{1}$ band to stain with Alcian Blue may be in some way related to the complexing of antibody with $\mathrm{SC}_{1}$ molecule.

Since the above evidence suggests that $\mathrm{SC}_{1}$ may be a mucopolysaccharide, as are the blood group substances, eluates from agar after electrophoresis were tested for the presence of $\mathrm{A}, \mathrm{B}, \mathrm{H}$, and $\mathrm{Le}^{\mathrm{a}}$ substances by hemagglutination inhibition. Eluates from the origin and somewhat anodal to the origin showed strong inhibition when the separated saliva was taken from a secretor, but no inhibition with non-secretor saliva. Similar results were obtained by Kinersly \& Leite (1957). Both secretors and non-secretors exhibited $\mathrm{SC}_{1}$ activity but only in eluates taken from an area of the gel $1-2 \mathrm{~cm}$. anodal to the $\mathrm{A}, \mathrm{B}, \mathrm{H}$, and Le $\mathrm{e}^{\mathrm{a}}$ substances. No inhibition could be detected with fractions containing $\mathrm{SC}_{1}$, and no $\mathrm{SC}_{1}$ could be detected by immunodiffusion in the $A, B, H$, and $L^{a}$ inhibiting fractions. These experiments elearly demonstrate that the $A B H$ and $\mathrm{L}_{\theta}$ blood specificities of saliva and the $\mathrm{SC}_{1}$ specificity are located on different molecules.

In order to explore the possibility of a relationship between $\mathrm{SC}_{1}$ level and P,T.c. threshold, fifty-six individuals were tested for both traits. P.T.c. thresholds were determined by the method of Harris \& Kalmus (1950). A positive but non-significant correlation $(r=0 \cdot 18)$ was found. It was therefore concluded that $\mathrm{SC}_{1}$ level and P.T.c. threshold are independent.

In the course of immuno-electrophoretic studies of parotid salivas, it was noted that $\mathrm{SC}_{1}$ concentration was extremely variable between subjects, ranging from no detectable precipitin band in some individuals to a very strong band in others. It was necessary to concentrate parotid saliva from certain individuals $20-25$ times to detect even a faint precipitin band, whereas other individuals gave strong bands with no concentration. The level of $\mathrm{SC}_{1}$ was also noted to be relatively constant and repeatable for different specimens from a given individual. Parotid saliva samples from ten pairs of identical twins indicated that monozygotic twins have similar levels of $\mathrm{SC}_{1}$, as judged by the strength of the precipitin band after immuno-electrophoresis of samples adjusted to similar total protein concentrations. Whereas parotid salivas showed gross variability in $\mathrm{SC}_{\mathbf{1}}$ concentration, the variation between whole saliva samples from different individuals was found to be less extreme. Not only is $\mathrm{SC}_{1}$ concentration less variable in whole saliva, but its concentration is relatively greater in the non-parotid secretions as evidenced by the fact that an $\mathrm{SC}_{1}$ band invariably formed when whole saliva was tested, even in cases where there was no detectable $\mathrm{SC}_{1}$ in the parotid saliva. However, since parotid secretion constitutes a large fraction of the whole saliva and is extremely variable in $\mathrm{SC}_{1}$ level, $\mathrm{SC}_{1}$ variation should also be detectable in whole saliva. As noted, $\mathrm{SC}_{1}$ is stable to boiling, therefore the samples collected for use in the several projects previously mentioned could be utilized for quantitative studies. In fact, since boiling precipitates all but one other minor component, analysis by immunodiffusion was greatly simplified. Although there is a probable loss in sensitivity for detection of $\mathrm{SC}_{1}$ variation accompanying the use of whole rather than parotid saliva, analysis was undertaken on whole saliva in the hope that the loss in sensitivity could be offset by the greater number of samples available to be classified. 
Preliminary testing indicated that variation in the whole salivas could be more readily detected by the immunodiffusion technique than by immuno-electrophoresis. Furthermore, the $\mathbf{S C}_{1}$ levels could be relatively accurately quantitated by measuring the position of the precipitin band on the immunodiffusion plate, as previously described. All subsequent discussion will be concerned with classification by this method. The relationship between precipitin band position and $\mathrm{SC}_{1}$ concentration is shown in Text-fig. 1 . The plotted relationship represents the regression line obtained from serial dilutions of twelve samples from the same subject. Also shown are the mean values for the twelve samples at each dilution.

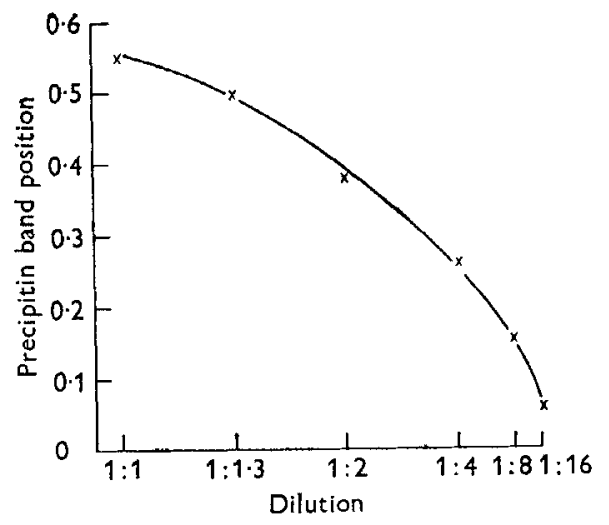

Texi-fig. 1. Regression line based on serial dilutions of twelve whole saliva samples from same individual. $x$, sample mean.

Before attempting analyses of genetic factors, the questions of the error of the method and the possibility of diurnal variation were investigated. Table 1 contains data from three analyses of variance which bear on these questions. Repeated tests were done of the same sample from each of six subjects. For four subjects, twelve repeats were done and, for the other two, ten trials each, giving a total of 68 observations. The results are presented in Table $1 a$. The 'within' mean square gives a minimum measure of the error inherent in the procedure and, as can be seen, is very much smaller than the mean square due to differences between individuals. Not included in the error estimate from this analysis are temporal changes in technique as well as the effects of different batches of agar. However, error from these sources is considered to be relatively small.

Two studies were conducted to test diurnal variation. First three individuals known to exhibit low, intermediate, and high levels of $\mathrm{SC}_{1}$ were selected. Ten samples were collected from each at different times (before and after meals, early morning, midday, evening, etc.) over a 3-week period. No correlation between time of day and $\mathrm{SC}_{1}$ level was noted, with the possible exception that samples from one individual, collected immediately on arising, tended to give much lower than average readings. Analysis of these data is given in Table 1b. As might be expected, the error associated with this procedure is considerably larger than for the preceding analysis, where the same samples were repeated. However, the difference between individuals appears highly significant. Since the three individuals tested in the foregoing were preselected on the basis of differing $\mathrm{SC}_{1}$ level, a similar experiment was carried out with an unselected sample of females. Three samples from each of forty-four women were tested. The results are given in Table $1 c$. Again there is a large difference between individuals. 
Table 1

(a) Analysis of variance-same sample replicated to or $\mathrm{r} 2$ times for each of six individuals

\begin{tabular}{lrccc}
\multicolumn{1}{c}{ Source } & D.F. & S.s. & M.s. & $\begin{array}{c}\text { Variance } \\
\text { ratio }\end{array}$ \\
Between individuals & 5 & 1.0285 & 0.2057 & 108.26 \\
Within & 62 & 0.1186 & 0.0019 & - \\
Total & 67 & I.I47I & $0.017 \mathrm{I}$ & -
\end{tabular}

(b) Means and analysis of variance-ten whole saliva samples from each of three individuals

\begin{tabular}{lcccc}
\multicolumn{1}{c}{ Source } & D.F. & s.s. & M.s. & $\begin{array}{c}\text { Variance } \\
\text { ratio }\end{array}$ \\
Between individuals & 2 & 0.4771 & 0.2386 & 17.94 \\
Within & 27 & 0.3588 & 0.0133 & - \\
Total & 29 & 0.8359 & 0.0288 & - \\
$\bar{X}_{1}=0.218$ & $\bar{X}_{2}=0.391$ & $\bar{X}_{8}=0.526$
\end{tabular}

(c) Analysis of variance-three whole saliva samples from each of 44 unselected individuals

\begin{tabular}{lrccc}
\multicolumn{1}{c}{ Source } & D.F. & s.s. & M.s. & $\begin{array}{c}\text { Variance } \\
\text { ratio }\end{array}$ \\
Between individuals & 43 & I.6084 & 0.0374 & 2.63 \\
Within & 88 & $1 \cdot 2509$ & 0.0142 & - \\
Total & I3I & 2.8593 & 0.0217 & -
\end{tabular}

After establishing that a considerably greater variation existed between individuals than for repeated samples from the same individual and that the error of the method was relatively small, an attempt was made to ascertain whether genetic factors play a part in the variability of $\mathrm{SC}_{1}$ concentration. The distribution of the values of relative precipitin band position, obtained for all unrelated individuals tested in this phase of the study, is shown in Text-fig. 2. These represent whole saliva samples from 296 subjects. These 296 values were obtained by randomly selecting one member from each twin and sib pair and one observation from each of the forty-four females. Of particular interest is the asymmetry of the distribution with an excess of observations at the lower end. The distribution is not normal when tested by chi-square. In spite of this departure from normality, the fit is considered sufficient to justify carrying out simple correlations and analyses of variance. These procedures are sufficiently robust that the results can be considered as reasonable approximations. However, little attempt has been made to attach definite probability estimates to the results.

The sexes are not equally represented in the groups making up this distribution; neither is age comparable between the groups. The forty-four non-twin sibs are all males less than 15 years of age, the forty-four females ranged in age from 20 to 35 years while the twins were 33 to $44-$ year-old males. The mean $\mathrm{SC}_{1}$ levels for the three groups were $0.340,0.380$, and 0.400 , respectively. The pooled variance is 0.0169 , in contrast to 0.0180 for the total variance. The difference between the means is significant by analysis of variance. If the means are tested pairwise, the females cannot be shown to be different from either of the other two groups. These findings suggest an age effect but no sex difference. The distributions of observations in the three groups are essentially similar in shape and have similar variances. This age effect is therefore of consequence only in slightly inflating the total variance and in perhaps slightly obscuring the detailed con- 
figuration of the curve. There is no indication that any of the groups is contributing disproportionately to the excess of low values seen in Text-fig. 2.

The distribution of intrapair differences in readings for twins and sibs is given in Table 2. It will be noted that fraternal twins and sibs both show several values higher than any seen for identical twins. For the comparison of identical and fraternal twins $\chi^{2}=9.884,0.025<P$ $<0.05$ (D.F. $=4$ ).

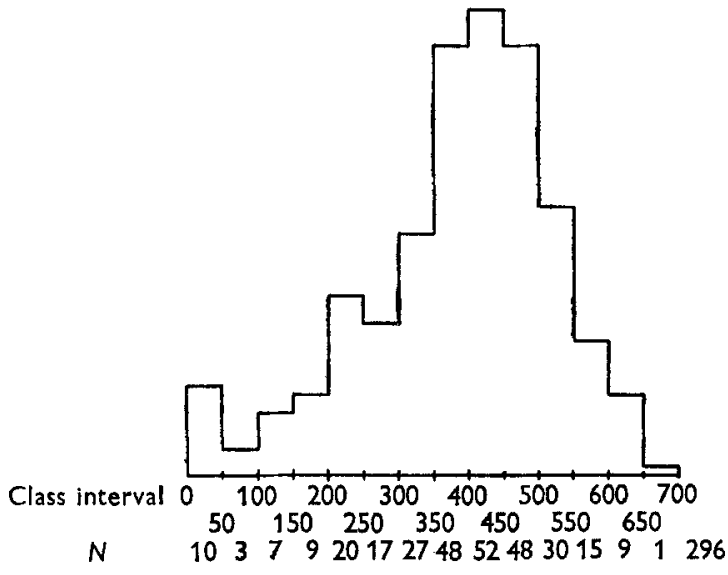

Text-fig. 2. Frequency distribution for 296 unrelated individuals. Number giving specified values for relative position of $\mathrm{SC}_{1}$ precipitin band $\bar{X}=0.390, S^{2}=0.018$.

Table 2. Frequency distribution-number of twin and sib pairs showing a specified difference in relative position of $S C_{1}$ precipitin band

\begin{tabular}{|c|c|c|c|}
\hline $\begin{array}{l}\text { Intrapair } \\
\text { difference }\end{array}$ & Monozygotic & Dizygotic & \\
\hline $0.000-0.050$ & 48 & 46 & \\
\hline $0.051-0.100$ & 25 & 20 & \\
\hline $0.101-0.150$ & 13 & I9 & \\
\hline $0.151-0.200$ & 5 & 7 & \\
\hline $0.201-0.250$ & 5 & I3) & \\
\hline $0.251 \cdots 0.300$ & - & $I$ & \\
\hline $0.301-0.350$ & 一 & 4 & \\
\hline $0.351-0.400$ & - & $6.2 \%$ & $9.1 \%$ \\
\hline $0.401-0.45^{\circ}$ & - & 0 & \\
\hline $0.45 I-0.500$ & - & ( & \\
\hline Total & 96 & $\operatorname{li2}$ & \\
\hline
\end{tabular}

$\mathrm{MZ}$ vs. $\mathrm{DZ} \chi^{2}=9.884 ; 0.025<P<0.05 ; \mathrm{D} . \mathrm{F} .=4 \cdot r_{\mathrm{MZ}}=0.7 \mathrm{I} \pm 0.05 \mathrm{I} ; r_{\mathrm{DZ}}=0.47 \pm 0.074 ; r_{\mathrm{sibs}}=$ $0.49 \pm 0.116$.

The within pair variances are 0.0041 and 0.0090 , respectively. For non-twin sib pairs the variance is 0.0088 . The variance ratio is $2 \cdot 195$, which with 109 and 95 D.F. is significant at $P<0.001$. While this probably represents a real difference, it is perhaps unwise to place too much reliance on this ratio in light of the deviation from normality of the distributions and the fact that the larger variance of the dizygotic twins is due primarily to a few very discrepant pairs.

Correlation coefficients were calculated for the twin pairs. Correlation between identical twins is $0.71 \pm 0.051$, for fraternal twins $0.47 \pm 0.074$, and for non-twin sibs $0.49 \pm 0.116$. All are signifi- 
cant at the $1 \%$ level; the difference between identical and fraternal is also significant. Again, because of the lack of normality in the data, these figures should be considered as approximations.

In an attempt to obtain more definitive evidence for genetic factors, it was decided to classify persons giving values of 0.250 or less as low $\mathrm{SC}_{1}$ secretors. Such values occurred with a frequency of about $15 \%$. Matings between two lows would be expected to occur about 1 in 50 times. One hundred and fifty matings, with two or more children, were screened before a low by low was found. All three offspring had low $\mathrm{SC}_{1}$ levels. The parents gave values of 0.081 and 0.125 , while the three children had $\mathrm{SC}_{1}$ levels of $0 \cdot 244,0 \cdot 136$, and $0 \cdot 120$.

\section{DISCUSSION}

The findings with specific antisera, together with absorption and inhibition studies, indicate that $\mathrm{SC}_{\mathbf{1}}$ is elaborated in the salivary glands and is not a serum component. Although usually present in parotid secretion, it occurs in relatively higher concentration in the non-parotid portion of saliva, suggesting an association with the mucus producing glands. Its resistance to denaturation and failure to react with a glycoprotein stain tend to argue against a simple protein or glycoprotein. While a firm conclusion is not possible, the foregoing, when considered together with the high electrophoretic mobility of the material, its insolubility in phenol-alcohol, and the staining of $\mathrm{SC}_{1}$ eluates with Alcian Blue, suggests that $\mathrm{SC}_{1}$ may be a mucopolysaccharide.

Evidence that genetic factors influence the $\mathrm{SC}_{1}$ level may be summarized as follows: (1) The correlations observed are consistent in that they are significantly larger for identical twins than for fraternal twins, whereas the sibs and fraternal twins give similar correlations. The relatively modest correlation between monozygotic twins suggests a large environmental component and/ or relatively large experimental error. The very small 'within' variance in Table $1 a$, relative to the 'within' variances in $1 b$ and $c$, suggests that much of the variation is due to environmental factors rather than experimental error.

It might be argued that the greater similarity in $\mathrm{SC}_{\mathbf{1}}$ levels of identical twins could be accounted for by more similar environments. However, all twins studied were adult males, for the most part married, with families, and living apart. It seems unlikely that the pertinent environmental factors should be much more similar for one group than the other. Also, one might expect the sib pairs, all of whom were children, to be subject to more uniform intrapair environmental conditions than the adult twins. (2) The low by low mating produced only low children. (3) There is an excess of low values seen in Text-fig. 2. There is some suggestion that these low values represent a second overlapping distribution, as might be expected on the basis of a single locus hypothesis. The fact that the intrapair differences for $6 \%$ of the $\mathrm{DZ}$ twins and $9 \%$ of the nontwin sib-pairs were greater than any difference observed for $M Z$ twins also suggests genetic factors.

Another small piece of evidence comes from the forty-four females. For this group, three samples from each individual were tested. If we were dealing with a variable distributed about a single mean, we might expect the variance of the forty-four means of three samples to be considerably smaller than the pooled estimate of $\sigma^{2}$ based on the total sample. The two estimates were found, in fact, to be quite similar, $S_{\bar{x}}^{2}=0.0124$ and $S^{2}=0.0169$. This finding implies a measure of reproducibility in successive samples from the same individual and suggests two or more overlapping classes, rather than a distribution about a single mean with large error. However, these classes could be environmental as well as genetic in origin. 
Although there is considerable evidence in favour of genetic control of $\mathrm{SC}_{1}$ level, the precise nature of this control is not clear. The present data are not inconsistent with control by a single locus but neither is more complex inheritance excluded. Evidence on which to base a firm conclusion is at present not available. Family studies using parotid specimens, more refined quantitative methods, and multiple specimens from each individual would probably help to clarify this situation.

\section{SUMMARY AND CONCLUSIONS}

Definite variation between individuals has been demonstrated in the concentration of a serologically detected component of saliva, which has electrophoretic mobility similar to serum albumin. Family data and evidence from twin studies indicate that this variation is genetically determined, but environmental modification appears relatively large. The data are insufficient for definite conclusions as to the exact nature of the genetic control. The component has been demonstrated to be absent from serum and, therefore, is probably elaborated in the salivary glands themselves. Limited evidence suggests that the component may be an acid mucopolysaccharide. It is not associated with the blood group substances.

This work was supported by U.S. Atomic Energy Commission Contract AT (11-1)-405. The authors wish to thank Dr Henry Gershowitz for many helpful discussions and suggestions, Dr George Solish, who made available the replicated female saliva samples, and Dr C. S. Chung, who kindly furnished saliva from individuals previously tested for P.T.c. threshold. The assistance of Dr James R. Hayward in obtaining parotid saliva by cannulation is gratefully acknowledged.

The School of Public Health Cardiovascular Research Center, through which the family specimens were obtained, is supported by Program Project Grant H-6378 from the National Heart Institute, National Institutes of Health.

\section{REFERENCES}

Curby, W. A. (1953). A device for collection of human parotid saliva. J. Lab. Clin. Med. 41, 493.

Crowle, AL. J. (1961). Immuno-diffusion, 333 pp. New York: Academic Press.

GABL, F. \& Pastner, D. (1960). Immunelektrophorese von speichelproteinen. Protides of the Biological Fluids, by H. Peeters (ed.). New York: Elsevier Publishing Co.

Grabar, P. (1959). Immunoelectrophoretic analysis. Meth. Biochem. Analy. 7, 1.

GrUBB, R. (1948). Correlation between Lewis blood group and secretor character in man. Nature, Lond., 162, 933.

Harris, H. \& Kalmus, H. (1950). The measurement of taste sensitivity to phenylthiourea (P.T.C.), Ann. Eugen., Lond., 15, 24-31.

Hayward, B. J. \& Augustine, R. (1957). Quantitative gel diffusion methods for assay of antigens and antibodies. Int. Arch. Allergy, N.Y., 11, 192.

Heremans, J. \& Vafrman, J. P. (1958). Différentiation sur papier des protéines et des mucopolysaccharides acides au moyen de colorations specifiques combinées. Clin. Chim. Acta, 3, 430.

Hirschresd, J. (1959). Individual precipitation patterns of normal rabbit sera. Acta Pathol. Microbiol. Scand. 46, 229.

Kabat, E. A. (1956), Blood Group Substances, 330 pp. New York: Academic Press Inc.

Kinersuy, T. \& LeITe, H. B. (1957). Paper electrophoresis study of some components of saliva. Yale J. Biol. Med. 29, 496.

Kostuin, AL. \& Rauch, S. (1957). Zur chemie des Ruhespeichels einzelner speicheldrusen. Helv. Med. Acta, 24, 600 .

Patton, J. R. \& Pigman, W. (1959). Electrophoretic and ultracentrifugal components of human salivary secretions. J. Amer. Chem. Soc. 81, 3055. 
Ridgway, G. J., Klontz, G. W. \& Matsumoto, C. (1962). Intraspecific differences in serum antigen of red salmon demonstrated by immunochemical methods. International North Pacific Fisheries Commission. Bulletin 8, page 1.

Schneyer, C. A. (1958). Genetic control of amylase levels of mouse submaxillary glands. Proc. Soc. Exp. Biol., N.Y. 98, 160.

Schiff, F. \& Sasaki, H. (1932). Der Ausscheidungstypus ein auf serologischem Wege nachweisbares mendelndes Merkmal. Klin. Wschr. 34, 1426-9.

URIEL, J. (1961). Caracterisation des cholinesterases ot d'autres esterases carboxyliques après electrophorese et immunoelectrophorese en gelose. Ann. Inst. Pasteur, 101, 104.

Weichselbaum, T. E. (1946). An accurate and rapid method for the determination of proteins in small amounts of blood serum and plasma. Amer. J. Clin. Path. 16, 40. 